\section{Model exercise}

\section{Brian Charlesworth}

\section{Theory of Natural Selection and}

Population Growth.

By Lev R. Ginzburg.

Benjamin-Cummings/Addison-Wesley: 1983. Pp.160. \$21.95, £16.95.

THERE are two approaches to mathematical modelling in biology: one is to seek general formulations of the properties of basic processes, without paying much attention to observational detail; the other is to try and produce models that explain particular classes of facts. Both the approaches are clearly needed for proper theoretical understanding (although most biologists would probably sympathize more strongly with the second), and the great pioneers of population genetics each contributed in both of these ways.

Lev Ginzburg's book is an example of the first approach. Although he covers a number of largely unrelated topics, ranging from the dynamics of complex ecosystems to the population genetics of multiallele and multi-locus systems, he is always concerned to extract generalizations about "macroparameters" from the jumble of underlying mechanisms. The book reads much more like Ginzburg's thoughts on a collection of topics than an attempt to survey a field, and I feel that he could, in places, have made greater acknowledgement of the work of others - for instance in relation to the stability of randomly chosen fitness sets in multiple allelic systems (Chapter 4), and the interaction of selection and density-dependence (Chapter 6).

Ginzburg's approach is frequently original and elegant, as exemplified by the treatment of change in mean fitness in multi-locus systems (Chapter 3). Here he demonstrates that a randomly-chosen $n$-dimensional vector of gamete frequency is expected to be located near the centre of the space of allowable frequency values, and uses this to show that mean fitness will usually increase in an $n$-locus system.

Of course, the biologist will ask what one can do with such generalizations, and the answer is often unclear. In the $\boldsymbol{n}$-locus case, for example, Ginzburg goes on to say that points in the neighbourhood of equilibria are not necessarily representative of randomly chosen points, so that his theorem does not rule out an important influence of recombination on the dynamics of change near equilibrium. My impression of this book is thus of a clever and enjoyable intellectual exercise but one which leaves me uncertain of its importance for evolutionary biology.

Brian Charlesworth is Reader in the School of Biological Sciences at the University of Sussex.

\section{More volcanology}

ELSEVIER'S new monograph series, Developments in Volcanology, has got off to a flying start with the recent publication of three hefty tomes with similarly illustrated hardback bindings. Interior style is rather less uniform, however, two of the books (Vols 1 and 3) being printed via camera-ready typescript and the other having been typeset in the more traditional way.

Although all three are edited "symposium" volumes, the origins of the material also differ. The first, Forecasting Volcanic Events (edited by H. Tazieff and J.C. Sabroux; pp.635, Dfl. 185, \$78.75), comprises 37 specially commissioned chapters all of which are science-based but some of which also deal with the social, legal and economic aspects of volcanic hazards. Indeed, the book is intended not just for volcanologists but also for nonscientists, particularly those in local government and administration who are so often vexed by the inability of volcanologists to come forward with clear-cut predictions unhedged by provisos. Readers who persevere will certainly understand the nature of scientific uncertainty by the end.

The second book, Arc Volcanism (edited by S. Aramaki and I. Kushiro; pp.652, Dfl. $230, \$ 97.75$ ), contains about $10 \%$ of the material presented at a 1981 conference and is a straight reprint of Vol.18 of the Journal of Volcanology and Geothermal Research. The 25 papers, all of which are specialist-scientific, range widely over the geochemistry (mainly), geology and geophysics of island-arc volcanic activity. Specialists will know what they are in for and the rest of us will give up in despair.

Explosive Volcanism (edited by M.F. Sheridan and F. Barberi; pp.481, Dfl. 220 $\$ 84.75$ ), also reprinted from $J$. Volcanol. geotherm. Res., comprises 20 papers resulting from a workshop held in 1982. Topics covered include the atmospheric hazards of volcanic activity, the past 5,000 years of activity at Mount Pelée, the littleknown AD 472 eruption of Vesuvius, the fundamentals of hydrovolcanism, the eruption of ignimbrites, volcanism in the Aleutian arc, computer-simulation of ash transport and deposition, and the evolution of the Fossa cone of Vulcano, as well as more detailed studies of the Sacrofano-Baccano caldera in Rome and the Somma-Vesuvius complex. Diverse though the topics are, the subject of explosive volcanism as a whole is rather more accessible than that of the previous volume and the text is thus less forbidding. Indeed, because a number of the articles treat the subject from the point of view of volcanic hazard, the book will be of interest to the natural audience for the first volume.

Peter J. Smith

\section{Cutting ecology down} to size

John H. Lawton

The Ecological Implications of Body

Size.

By Robert Henry Peters.

Cambridge University Press: 1983.

Pp.329. £17.50, \$29.95.

How much does a rhinoceros eat? What would be a good estimate for the defaecation rate of a brontosaurus? What is the expected ratio of biomass for krill and whales in the Antarctic Ocean? Ecologists are often confronted by questions of this kind, but lacking the necessary data or the means of obtaining them have to guess the answers using reasonable assumptions. Undoubtably "guestimates" are much improved if we know the sizes of the organisms involved, because there exists a rich set of relationships between the rate at which individual animals do things and their body size. These allometric relationships are the subject of Peters's book.

All biologists know of the existence of some allometric relationships, one of the most familiar being the " $3 / 4$ power rule" for metabolic rates - that is, to a good approximation, individual metabolic rates for different species scale as the 0.75 power of their body weights, with three broad groups of animals (unicells, poikilotherms and homoiotherms) differing only in the intercept of the underlying relationship, not in its slope. But such relationships, most of them less well known, exist for many other animal attributes. By describing a large number of body-size relationships in standard form, Peters has performed an invaluable service both for ecologists and for physiologists. Sixty pages of appendices summarize hundreds of empirically determined equations linking body size to metabolic rates for various taxa under different conditions of temperature and activity, to physiological variables such as pulse rate or total sleep time, to costs of movement, to water and nutrient requirement, to home range size and so on. Here is a treasure house of information.

The bulk of the text is a lucid general account of the ecological utility of this information, commencing with conventional problems in what might broadly be termed physiological ecology. Good examples are the capacity of birds and mammals of different sizes to withstand low temperatures, and the contrasting costs of migration in animals that have to fly, swim or walk to their destination. This material occupies Chapters 3-6, following an introduction and a mathematical primer on such matters as logarithms and power functions in Chapter 2. The physiological theme resurfaces in Chapter 13 where Peters reviews explanations for why, in 